EUROPHYSICS LETTERS

?? 2000

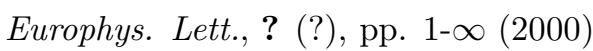

\title{
Motion of an object through a dilute quantum fluid
}

\author{
T. WinieCKi and C. S. AdAms \\ Department of Physics, University of Durham, Rochester Building, South Road, Durham, \\ DH1 3LE, England. UK.
}

(received ; accepted )

PACS. 03.75.F - Bose-Einstein condensation.

PACS. 47.37.+q- Superfluid hydrodynamics.

PACS. 67.40.Vs - Vorticity in ${ }^{4} \mathrm{He}$.

\begin{abstract}
. -
We simulate the motion of a massive object through a dilute Bose-Einstein condensate by numerical solution of the non-linear Schrödinger equation coupled to an equation of motion for the object. Under a constant applied force, the object accelerates up to a maximum velocity where a vortex ring is formed which slows the object down. If the applied force is less than a critical value, the object becomes trapped within the vortex core. We show that the motion can be described using the time-independent quantum states, and use these states to predict the conditions required for vortex scattering.
\end{abstract}

\section{Introduction. -}

One of the most elementary questions that can be asked about a fluid, is how will an object move through it? In quantum fluids, it is expected that the object moves without resistance at velocities, $v$, up to a critical value, $v_{\mathrm{c}}$, where energy and momentum conservation allow excitations. If the object mass is large, the critical velocity is given by the Landau criterion, $v_{\mathrm{c}}=(\epsilon / p)_{\min }$, where $\epsilon$ and $p$ are the energy and momentum of the excitation [1]. Experiments on the motion of objects in liquid helium suggest that the excitations are often vortices [2, however, understanding the exact mechanism of vortex formation is impeded by the lack of a complete hydrodynamical model. In contrast, for dilute quantum fluids such as the recently discovered atomic vapour Bose-Einstein condensates [3], the dynamics can be accurately described by the Gross-Pitaevskii equation, a form of non-linear Schrödinger equation (NLSE) [ 4 . Consequently, this system provides a near ideal testing ground for advancing our knowledge of superfluid flow. Experimental measurements of the heating produced by laser beam moving through an atomic condensate suggest that the dominant mechanism of momentum transfer is vortex shedding [5], in agreement with NLSE simulations of a two-dimensional homogeneous flow past a fixed object [6, 4, 8].

However, if the object has a finite mass, the fluid back-action is significant, and completely different dynamics can arise. For example, ions in liquid helium nucleate vortex rings and become trapped within the vortex core [2, 9]. Here, we study the general case of the motion 
of an object with finite mass moving through a dilute Bose-Einstein condensate. The timeevolution is found by solving a NLSE coupled to an equation of motion for the object. We show that the object can be accelerated up to a maximum velocity where a vortex ring emerges encircling the object. If the applied force is not too large, the object subsequently becomes trapped within the core of the ring. We show that the motion can be predicted from the time-independent uniform flow states and apply this method to give a complete description of energy and momentum conservation during vortex scattering.

\section{Numerical method. -}

Throughout the paper we use dimensionless units, where distance and velocity are measured in terms of the fluid healing length, $\xi$ and the speed of sound, $c$, respectively. The dynamics of the fluid are described by a wavefunction, $\psi(\boldsymbol{r}, t)$, whose time-evolution in the fluid rest frame is given by

$$
\mathrm{i} \partial_{t} \psi(\boldsymbol{r}, t)=-\frac{1}{2} \nabla^{2} \psi(\boldsymbol{r}, t)+V(\boldsymbol{r}-\boldsymbol{v} t) \psi(\boldsymbol{r}, t)+|\psi(\boldsymbol{r}, t)|^{2} \psi(\boldsymbol{r}, t) .
$$

where $V$ is the object potential. The position of the object is given by

$$
M \dot{\boldsymbol{v}}=\boldsymbol{F}+\int \mathrm{d}^{3} r \frac{\mathrm{d} V}{\mathrm{~d} \boldsymbol{r}}|\psi(\boldsymbol{r}, t)|^{2},
$$

where $\boldsymbol{F}$ is an external force and the second term is the force on the object due to the fluid. The computation is simplified by transforming into the frame of the object, where Eq. (1) may be rewritten as

$$
\mathrm{i} \partial_{t} \tilde{\psi}\left(\boldsymbol{r}^{\prime}, t\right)=-\frac{1}{2} \nabla^{2} \tilde{\psi}\left(\boldsymbol{r}^{\prime}, t\right)+V\left(\boldsymbol{r}^{\prime}\right) \tilde{\psi}\left(\boldsymbol{r}^{\prime}, t\right)+\left|\tilde{\psi}\left(\boldsymbol{r}^{\prime}, t\right)\right|^{2} \tilde{\psi}\left(\boldsymbol{r}^{\prime}, t\right)+\mathrm{i} \boldsymbol{v} \nabla^{\prime} \tilde{\psi}\left(\boldsymbol{r}^{\prime}, t\right),
$$

where $\tilde{\psi}\left(\boldsymbol{r}^{\prime}, t\right)=\psi(\boldsymbol{r}, t)$ is the wavefunction in the fluid frame written in terms of the object frame coordinates, $\boldsymbol{r}^{\prime}=\boldsymbol{r}-\boldsymbol{v} t$. The system is prepared in a time-independent laminar flow state, $\tilde{\psi}\left(\boldsymbol{r}^{\prime}, t\right)=\phi\left(\boldsymbol{r}^{\prime}\right) \mathrm{e}^{\mathrm{i} \mu t}$, where $\mu$ is the chemical potential, by solving Eq. (3) using Newton's method [10, 11]. From this initial state, the time evolution due to an applied force, $\boldsymbol{F}$, is evaluated by integrating Eq. (3) using a semi-implicit Crank-Nicholson formula. The conservation of momentum

$$
\boldsymbol{P}_{0}+\boldsymbol{F} t=M \boldsymbol{v}+\frac{\mathrm{i}}{2} \int \mathrm{d}^{3} r^{\prime}\left[\psi \nabla^{\prime} \psi^{*}-\psi^{*} \nabla^{\prime} \psi\right],
$$

where $\boldsymbol{P}_{0}$ is the initial momentum of the fluid, is imposed as an additional constraint. The equations are discretized on a three dimensional grid using the non-linear transform $\hat{x}=$ $x /(D+|x|)$, where $D$ is a scaling parameter, to map an infinite box onto the space $-1 \rightarrow 1$. The grid contains 140 points in each dimension and we use a time step $\mathrm{d} t=0.02$. For the object we choose a penetrable sphere with mass $M=200$ and radius $R=3.3(V=1.0$ for $\left|\boldsymbol{r}^{\prime}\right| \leq 3.3$ and 0 elsewhere). For an atomic condensate this would correspond to a small cluster of impurity atoms in a sphere with a diameter of a few microns. However, the main conclusions are not overly sensitive to these parameters.

Results. -

The evolution of the object velocity, $v$, due to a constant applied force, $F$, is shown in Fig. 1. As the velocity increases, momentum is transferred from the object to the fluid. This momentum transfer can be described in terms of an increase in the effective or hydrodynamical mass of the object, $m_{\mathrm{eff}}=(\partial P / \partial v)^{-1}$, where $P=F t$. The effective mass becomes infinite at the critical velocity, $v_{\mathrm{c}}=0.68$, and then negative as the object begins to slow down. Further insight is provided by the surface contour plots of the fluid density shown in Fig. 2. At the 
peak velocity, $v_{\mathrm{c}}$, a vortex ring emerges encircling the object. The object is weakly bound in the direction of motion, therefore as the ring grows, the object and the ring decelerate.

(a)

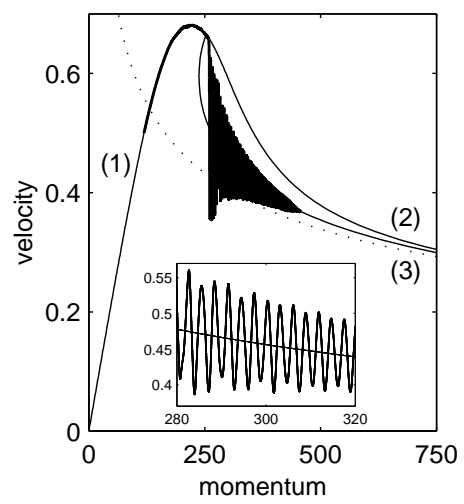

(b)

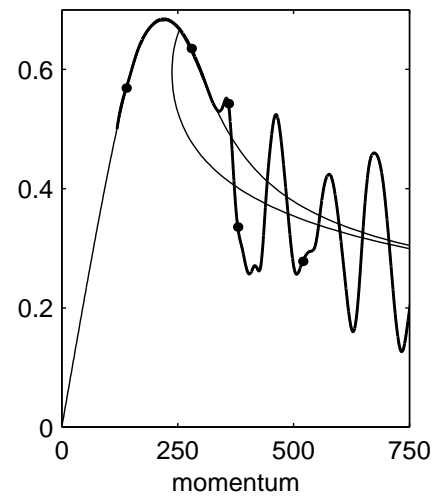

(c)

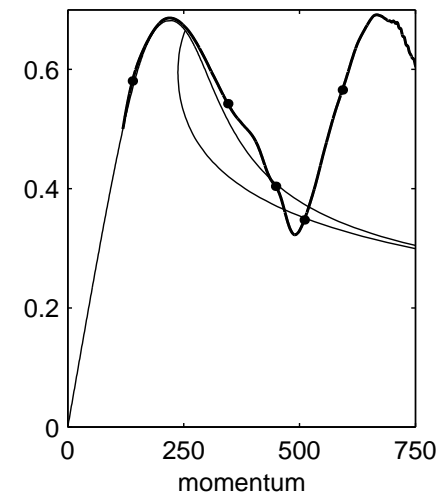

Fig. 1. - The evolution of the object velocity due to a constant applied force. The velocity is plotted (bold line) against the total momentum $\boldsymbol{P}=\boldsymbol{F} t$ for (a) $\boldsymbol{F}=0.05$, (b) $\boldsymbol{F}=2$ and (c) $\boldsymbol{F}=4$. Also shown are the time-independent solutions of the uniform flow equation, Eq. (3), corresponding to (1) laminar flow, (2) an encircling vortex ring, and (3) a pinned ring. As the object accelerates momentum is transferred from the object to the fluid. At the peak velocity, $v_{\mathrm{c}}=0.68$, an encircling vortex ring emerges and begins to slow the object down. An abrupt decrease in velocity occurs when the object jumps into the vortex core. This jump excites vibrations of the ring leading to large oscillations of the object velocity (inset). The oscillations are damped by the emission of sound waves. In (c), the force is sufficient to detach the object from the ring and the cycle repeats.

When the vortex core begins to separate from the object boundary, the encircling ring configuration becomes unstable with respect to transverse motion, and stochastic fluctuations induce a transition to a pinned ring, where the object is bound within the vortex core (see Fig. 2). In our simulations, defining the external force, $\boldsymbol{F}$, at a slight angle to the numerical grid axis is sufficient to induce the transition. On moving into the core, the object acquires a transverse velocity thereby deflecting its trajectory (Fig. 21). The deflection angle is a few degrees so this effect could be observable. If the ring detaches, a second ring forms and the object is pulled back in the opposite direction. Consequently vortices are emitted on alternating sides of the object, similar to the vortex shedding behaviour observed in classical fluids.

The jump into the core also leads to the excitation of oscillatory modes of the vortex ring Fig. 2(a). One mode of oscillation dominates [12] and the frequency is independent of the applied force. As the fluid is compressible, an accelerating object creates sound waves which damp the motion. This damping is apparent in the oscillations of the object velocity in Fig. 1 (a) inset. If the applied force is maintained the vortex radius continues to increase and eventually the motion becomes indistinguishable from that of a free vortex ring, indicated by the dotted line in Fig. 1 (a). Also shown in Fig. 1 1 are velocity-momentum curves predicted by the time-independent solutions of the NLSE for a uniform flow, Eq. (3). The three branches correspond to (1) laminar flow, (2) an encircling vortex ring and (3) a pinned vortex ring [11. Excluding the ring excitations, the motion closely follows the time-independent states. It follows that appropriate uniform flow solutions may be used to predict the motion of more 
(a)
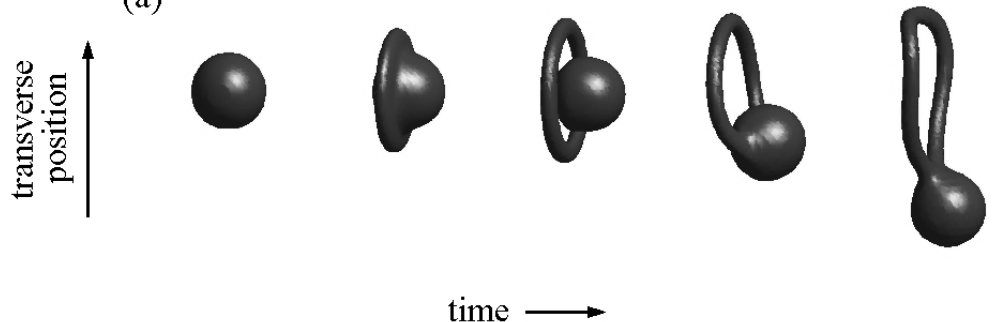

time

(b)
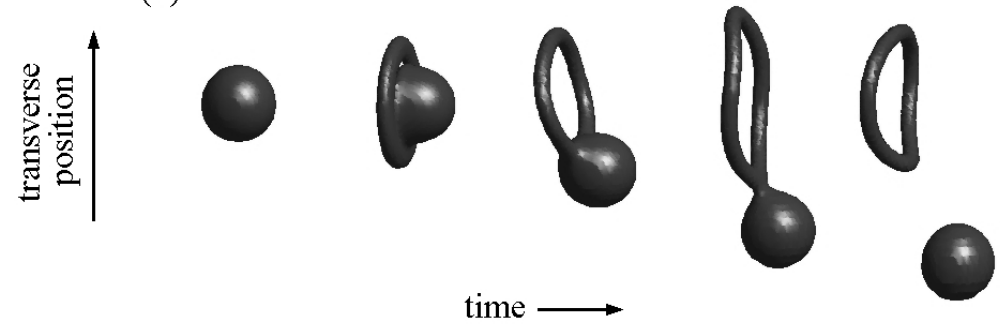

Fig. 2. - Sequence of surface contour plots of the fluid density for (a) $\boldsymbol{F}=2$ and (b) $\boldsymbol{F}=4$. The motion is from left to right, and the real space deflection due to the attraction of the vortex core is indicated by the $y$ coordinate. The momentum (or time) of each frame is indicated by a dot in Fig. 1(b) and (c), except for the last frame in (a), where $P=1562$. Note that after detachment, (b), the ring size remains constant and the object and ring move at different velocities.

complicated objects. To test whether a spherical object favours the encircling vortex ring configuration, we performed calculations on a sphere $(R=3.3)$ with a hemispherical surface bump $(R=1.5)$. The largest effect occurs when the bump lies in the equatorial plane. In this case, the critical velocity is reduced from 0.68 to 0.65 , and the vortex ring emerges asymmetrically with its axis pulled towards the bump. However, the initial ring radius is still similar to the no bump case. Subsequently, the object or ring rotate such that the vortex core is pinned to the bump.

Vortex detachment. -

If the applied force is larger, the object can detach from the vortex ring as in Fig 2(b). After detachment the size of the ring remains constant, and the object can accelerate again up to the critical velocity where another ring forms and so on. The beginning of this repetitive cycle is apparent in the time evolution shown in Fig. 1(c). If detachment occurs, the initial encircling or pinned ring system evolves into an object and a free vortex ring which moves more slowly as in Fig. 2(b). The nucleated ring can be considered as an excitation of the fluid with energy $\epsilon$ and momentum $p$. The dispersion curve for vortex rings in the NLSE was determined by Jones and Roberts 13 and is reproduced in Fig. 3. The simple form of the Landau criterion, $v_{\mathrm{c}}=(\epsilon / p)_{\min }$, cannot be applied to unbounded fluid as the minimum occurs in the limit $p \rightarrow \infty$. A complete description of energy and momentum conservation during vortex scattering should include the recoil energy $p^{2} / 2 M$, and the energy and momentum stored in the laminar flow around the object. Such a description is provided by considering the time-independent quantum states. In this picture, the process of vortex scattering appears as a 'decay' of an encircling ring state into a free vortex ring and a laminar flow state. In 
Fig. 3, we plot the dispersion curves for a moving object and a free vortex ring obtained from the time-independent solutions [11]. The shaded region indicates the range of initial energies and momenta where vortex scattering may occur, i.e., for which there exists final vortex ring and laminar states which satisfy the conservation laws. In a time-dependent simulation with a constant external force, the object moves up the dispersion curve passing through the critical point where a vortex is formed and then continues along the encircling ring branch (2). When the system enters the shaded region, (point A in Fig. 3), energy and momentum conservation permit decay into an object dressed by laminar flow (B) and a free vortex ring (C). Note that the energy at point $\mathrm{A}$ is higher than the time-independent value because the object is dragged out of the plane of the ring (see Fig 2). If the applied force is low, the object moves into the vortex core and the system relaxes towards the lower branch of the dispersion curve (3) making detachment unlikely.

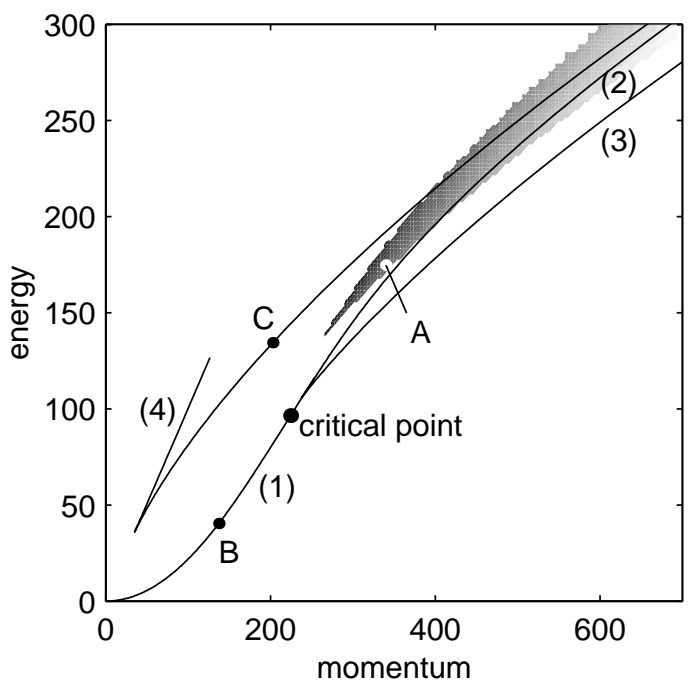

Fig. 3. - Energy and momentum conservation during vortex scattering. The allowed energy and momentum states of a moving object with mass $M=200$ (1-3) and a free vortex ring (4) determined from the time-independent solutions of the uniform flow equation, Eq. (3). Under a constant force, the object moves up the laminar flow branch of the dispersion curve (1) passing through the critical point where a vortex ring is formed, then continues along or just above the encircling ring branch (2). When the system reaches the shaded region, it is possible for the bound object-vortex ring (A) to decay into an object dressed by a laminar flow (B) and a free vortex ring (C). If, before detachment, the object moves into the core, the energy tends towards the pinned ring branch (3) making detachment less probable. The grey scale indicates the radius of the scattered vortex ring (darker is smaller). The velocity is determined by the slope of the dispersion curves.

Discussion. -

It is interesting to note that the velocity profile shown in Fig. 1(a) is quite similar to those observed for ions in superfluid helium (see e.g. [14]). To convert between dimensionless units and values for helium, we use the measured values of the number density, $n_{0}=2.18 \times 10^{28} \mathrm{~m}^{-3}$, the quantum of circulation, $\kappa=h / m=9.98 \times 10^{-8} \mathrm{~m}^{2} \mathrm{~s}^{-1}$, and the healing length $\xi / \sqrt{2}=$ $0.128 \mathrm{~nm}$ [9] leading to a mass unit, $\tilde{m}=m n_{0} \xi^{3}=0.13 m$, where $m$ is the mass of a helium 
atom. Our object parameters, $R=3.3, M=200$, correspond to 25 helium atoms with radius $0.6 \mathrm{~nm}$, similar to the 'snowball' that surrounds a positive ion [2]. In the NLSE model, the speed of sound is given by $c=\hbar / m \xi$, therefore using the healing length as the fit parameter, one obtains $v_{\mathrm{c}} \sim 60 \mathrm{~ms}^{-1}$, in rough agreement with experiments 14. Although the NLSE cannot be expected to give a complete description of superfluid ${ }^{4} \mathrm{He}$, it could be considered to compliment existing theories of vortex formation by ions [15], because the vortex core structure is included explicitly.

\section{Summary. -}

In summary, we have studied the motion of an object through a dilute quantum fluid. We show that there is a continuous transition between laminar flow and an encircling ring followed by a jump where the object moves into the vortex core. This jump leads to a deflection of the object trajectory and excitations of the vortex ring. If the object has a surface bump near the equator, the encircling vortex emerges asymmetrically. If the applied force is large, the object evades capture by the ring leading to periodic vortex shedding. The simulations reproduce the velocity evolution observed for ions in superfluid helium, suggesting that the behaviour may be characteristic of more complex quantum fluids. We show that the motion and the conditions required for vortex scattering can be predicted using the time-independent states for uniform flow. This approach could be extended to provide useful insight into other complex problems in quantum fluid mechanics such as vortex reconnections and sound emission.

We thank D. C. Samuels, C. F. Barenghi, B. Jackson and J. F. McCann for stimulating discussions. Financial support for this work is provided by the Engineering and Physical Sciences Research Council (EPSRC).

\section{REFERENCES}

[1] D. R. Tilley and J. Tilley, Superfluidity and superconductivity, 3rd Ed., (IoP, Bristol, 1990).

[2] R. J. Donnelly, Quantized vortices in Helium II, (CUP, Cambridge, 1991).

[3] See Bose-Einstein condensation in atomic gases, Proc. Int. School of Physics Enrico Fermi, eds. M. Inguscio, S. Stringari and C. Wieman (IOS Press, Amsterdam, 1999).

[4] F. Dalfovo, S. Giorgini, L. P. Pitaevskii, and S. Stringari, Rev. Mod. Phys. 71, 463-512 (1999).

[5] C. Raman, M. Köhl, R. Onofrio, D. S. Durfee, C. E. Kuklewicz, Z. Hadzibabic, and W. Ketterle, Phys. Rev. Lett. 83, 2502-2505 (1999).

[6] T. Frisch, Y. Pomeau, and S. Rica, Phys. Rev. Lett. 69, 1644-1647 (1992).

[7] T. Winiecki, J. F. McCann, and C. S. Adams, Phys. Rev. Lett. 82, 5186-5189 (1999).

[8] T. Winiecki, B. Jackson, J. F. McCann, and C. S. Adams, J. Phys. B. 33, to appear (2000).

[9] G. W. Rayfield and F. Reif, Phys. Rev. 136, 1194-1208 (1964).

[10] C. Huepe and M.-É. Brachet, C. R. Acad. Sci. Paris, 325, Série 2 b, 195-202 (1997).

[11] T. Winiecki, J. F. McCann, and C. S. Adams, Europhys. Lett. 48, 475-481 (1999).

[12] D. C. Samuels and R. J. Donnelly, Phys. Rev. Lett. 67, 2505-2508 (1991).

[13] C. A. Jones and P. H. Roberts, J. Phys. A 15, 2599-2619 (1982).

[14] G. W. Rayfield, Phys. Rev. Lett. 19, 1371-1373 (1967).

[15] C. M. Muirhead, W. F. Vinen, and R. J. Donnelly, Phil. Trans. R. Soc. Lond. A 311, 433-467 (1984). 